\title{
A 3 tesla magnetic resonance imaging volumetric analysis of the hippocampal formation: dependence on handedness and age
}

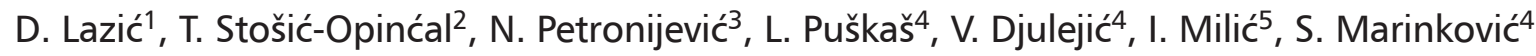 \\ ${ }^{1}$ Psychiatric Clinic "Laza Lazarević," Faculty of Medicine, University of Belgrade, Belgrade, Serbia \\ ${ }^{2}$ Department of Radiology, Institute of Radiology, Faculty of Medicine, University of Belgrade, Belgrade, Serbia \\ 3 Institute of Biochemistry, Faculty of Medicine, University of Belgrade, Belgrade, Serbia \\ ${ }^{4}$ Institute of Anatomy, Faculty of Medicine, University of Belgrade, Belgrade, Serbia \\ ${ }^{5}$ Clinic of Neurosurgery, Clinical Centre of Serbia, Faculty of Medicine, University of Belgrade, Belgrade, Serbia
}

[Received 10 January 2015; Accepted 15 February 2015]

Background: The hippocampal formation (HF) is one of the most important parts of the brain in the magnetic resonance imaging (MRI) volumetric analysis in various domains, but not completely from all aspects, including the handedness. The aim of our study was to evaluate the possible differences in the volume of the right and left HF among the healthy right-handed and left-handed subjects, and to determine whether the volume differences are age related.

Materials and methods: The MRI of this prospective study was performed using T1 fast field echo (FFE) sequence. The 124 subsequent coronal slices (thickness $1.5 \mathrm{~mm}$ ) were performed in each participant. The obtained HF volumes were normalised and statistically compared. Volunteers comprised 30 persons aged 22.0 years, 12 of whom were the left-handed, and 30 persons aged 75.2 years on average, 9 of whom were the left-handed.

Results: The right and left HF volumes averaged $2.986 \mathrm{~cm}^{3}$ and $2.858 \mathrm{~cm}^{3}$ in the right-handed, and $2.879 \mathrm{~cm}^{3}$ and $3.020 \mathrm{~cm}^{3}$ in the left-handed young volunteers, as well as $2.728 \mathrm{~cm}^{3}$ and $2.650 \mathrm{~cm}^{3}$ in the right-handed, and $2.617 \mathrm{~cm}^{3}$ and $2.780 \mathrm{~cm}^{3}$ in the left-handed elderly persons. The HF volume ratios in the young left-handed participants showed a significant left-greater-than-right asymmetry. A significant difference was also noticed within the right-to-left volume ratios of the right- and left-handed young and elderly participants. The latter reduction in the HF volume within the aged group can be interpreted as a slight atrophy of the HF. Conclusions: There is a significant difference in the volumes of the left and right HF of the left-handed young participants. The age related HF volume differences were proven between the groups of the young and elderly volunteers. The obtained data should be included into the future MRI studies of the HF volumes in various clinical domains. (Folia Morphol 2015; 74, 4: 421-427)

Key words: aging, dentate gyrus, gender, handedness, hippocampus, magnetic resonance, volumetric analysis

Address for correspondence: S. Marinković, MD, PhD, Institute of Anatomy, Faculty of Medicine, University of Belgrade, Subotić 4/2, 11000 Belgrade, Serbia, tel: +381-11-2645958, fax: +381-11-2686172, e-mail: mocamarinkovic@med.bg.ac.rs 


\section{INTRODUCTION}

The medial structures of the temporal lobe, especially the hippocampal formation (HF), play an important role in performance of the complex cognitive and behavioural tasks, such as the learning process, memory formation, language, planning, and processing of some emotions [9, 19, 23, 35]. Malformation, disorder or damage of the HF have a great importance in the pathogenesis, or consequences, of the variety of the neurological and psychiatric disorders, e.g. the epilepsy, schizophrenia, amnesia, depression, anxiety, posttraumatic stress, aging, Alzheimer's and Parkinson's disease [14, 26, 37, 38, 41, 42, 49]. Even the slightest volume changes, which can be identified by modern imaging techniques, may indicate certain neuropathology or pathophysiology in this region. Therefore, many of the mentioned authors use the magnetic resonance imaging (MRI) volumetric analysis of the HF in the following-up or determining the prognosis of the mentioned psychiatric and neurological disorders.

The volume of the HF depends, among other things, on the subjects' handedness, i.e. on the lateralisation of certain functions in the cerebral hemispheres [30, $41,44]$, as well as on the age of the examined patients $[2,8,16,30,33]$. Some studies indicated that certain cognitive functions or the particular neurological diseases affecting the HF have preference for the left or the right hippocampus of either the left- or right-handed persons, in whom they usually cause a reduction of the grey matter $[10,20,28,29]$. The neuroanatomical imaging studies showed that changes in the HF's amount is age related, but its extent is always less than in certain neurodegenerative disorders [34]. Furthermore, the incidence of some diseases that affect the $\mathrm{HF}$ parenchyma is higher among elders, including those with Alzheimer disease.

All in all, there are only a few reports dealing, at least partially, with the 3 parameters mentioned in our study, i.e. the age, handedness and gender $[2,8,29,44]$. Accordingly, the aim of this study was to determine the possible differences in the volumes of the right and left hippocampal formation among healthy right-handed and left-handed subjects of both sexes, and to determine whether those differences are age related.

\section{MATERIALS AND METHODS}

Our study included 60 healthy volunteers divided into two groups. The first group, with a mean age of
$22.0 \pm 2.5$ years, consisted of 30 students of the Medical Faculty, 12 of whom were the left-handed (40\%) and the remaining the right-handed ones (60\%). The second group included 30 elderly persons, averaged age $75.2 \pm 1.8$, without the neurological signs and symptoms, and with the normal magnetic resonance (MR) brain images. Within this group, 9 (30\%) were the left-handed individuals. Written consent was obtained from each participant, and the whole study was approved by the Ethics Committee of the University Clinical Centre.

The MRI examinations took place in the Railway Workers Health Care Institute in the period from March to July 2013. The MRI was performed in the 3T Philips Intera MRI scanner using a sensitivity encoding (SENSE) head coil. In order to evaluate the brain structures and to exclude gross pathology, the whole brain images in coronal plane were obtained (total 124 scans per person) using T1-weighted fast field echo (FFE) sequence [field of view $25.0 \times 17.5$ $\mathrm{cm}$, matrix $512 \times 512$, repetition time (TR) $9 \mathrm{~ms}$, echo time (TE) $1.9 \mathrm{~ms}$, inversion time (TI) $500 \mathrm{~ms}$, flip angle $20^{\circ}$, slice thickness $1.5 \mathrm{~mm}$, gap $0 \mathrm{~mm}$ ].

A morphometric study of the obtained coronal images was performed using semiautomatic commercially available software (Philips Intera software package). Regions of interest were established according to a previously defined protocol [4, 16]. The anterior border of the HF emerged beneath the amygdala, i.e. in a section that demonstrates the mammillary bodies. We chose as a posterior boundary a section through the caudal part of the pineal body. The semiautomatic procedure included the sequential reading, contrast enhancing, and manual delineation of the structures, as well the automatic calculation of their cross-sectional area. In order to eliminate the individual variation in the intracranial volume among the subjects $[3,4,11,25$, 36], data regarding the individual variables in each person were normalised according to the following formula [7]:

$$
C V_{r}=\frac{T C V}{T C r V} \cdot 100
$$

The values represent the relative cerebral volume $(\mathrm{CVr})$, the total cerebral volume (TCV) and the total intracranial volume (TCrV), respectively. The obtained absolute HF volume values were multiplied with $\mathrm{CVr}$ and thus transformed into the normalised $\mathrm{HF}$ volumes. The provided data were analysed using the 
SPSS 12 software program. The statistical parameters included a standard deviation ( $\pm S D$ ), " $t$ ", "DF", and " $p$ " values $(p<$ or $>0.05)$.

Finally, in order to illustrate the HF from the anatomical and histological aspect, we made a coronal section through the $\mathrm{HF}$ of a brain and head injected with and fixed in a $10 \%$ formaldehyde solution for 6 weeks. In addition, a piece of the HF was prepared for a standard histological examination, and then stained with cresyl violet (Nissl method).

\section{RESULTS}

The HF is a specific part of the medial portion of the temporal lobe which belongs to the limbic system. The HF mainly comprises the hippocampus and the dentate gyrus [6], which are characteristically rolled in upon themselves, just medial and above the parahippocampal gyrus, and which extends along the floor of the inferior horn of the lateral ventricle (Fig. 1). The histological examination shows a large number of the pyramidal neurons within the hippocampus, and a smaller number of the neurons forming the dentate gyrus (Fig. 2).

The largest part of our study is referred to the MRI examination of the HF volume (Figs. 3A, B). The results of the measuring and normalisation of the $\mathrm{HF}$ volume in the MRI, which are classified in relation to the handedness and age, are presented for both the right and left hemispheres of the two mentioned groups of the volunteers of both (Table 1).

The obtained data showed, firstly, that in the right-handed individuals of both groups the right HF was slightly larger than the left one and, similarly, in the left-handed individuals of the two groups the left HF was slightly larger than the opposite one (Table 1). However, a statistical significance of the obtained data was not confirmed.

Secondly, data within the first group, i.e. those related to the young left-handed volunteers, presented a significant difference $(t=2.35 ; D F=11 ; p<0.05)$ between the HF volumes ratios of the left and right cerebral hemispheres. On the other hand, the analysis did not reveal a significant difference related to the side of the HF volume in the young right-handed volunteers. In other words, the values did not differ significantly between the two hemispheres $(t=10.34 ; D F=17 ; p>0.05)$. However, the right-to-left $\mathrm{HF}$ volume ratios differed significantly between the right- and left-handed participants $(p<0.05)$, but only in the left hemisphere.

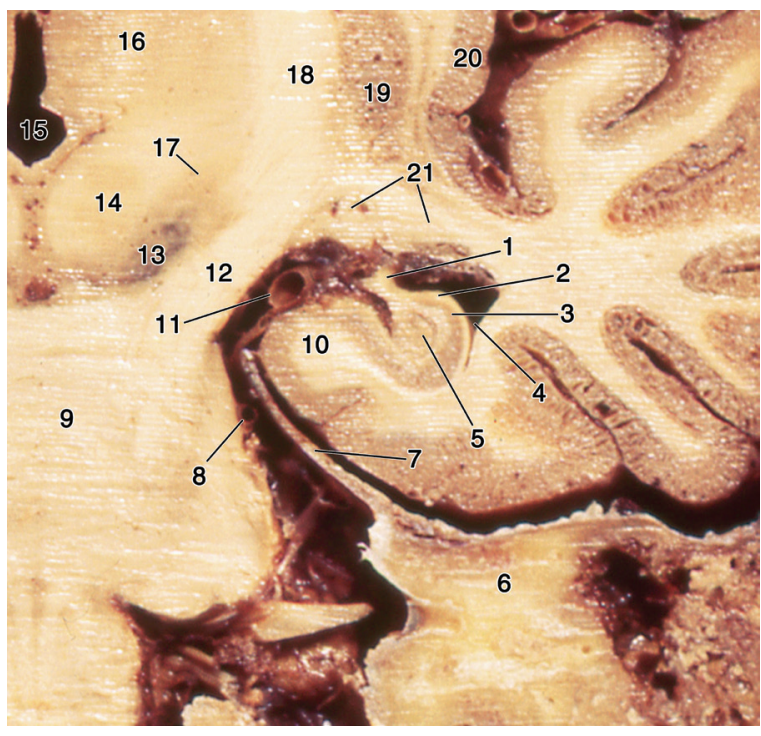

Figure 1. Coronal section of the left hippocampal formation; 1 - the fimbria of the hippocampus; 2 - the alveus; 3 - the hippocampus; 4 - the inferior horn of the lateral ventricle; 5 - the dentate gyrus; 6 - the petrous part of the temporal bone; 7 - the tentorium; 8 the superior cerebellar artery (cut); 9 - the base of the pons; 10 the parahippocampal gyrus; 11 - the P2 segment of the posterior cerebral artery; 12 - the cerebral peduncle; 13 - the substantia nigra; 14 — the red nucleus; 15 - the 3rd ventricle; 16 - the thalamus; 17 - the subthalamic nucleus; 18 - the posterior limb of the internal capsule; 19 - the putamen; 20 - the insular cortex; 21 - the medial and lateral geniculate bodies.

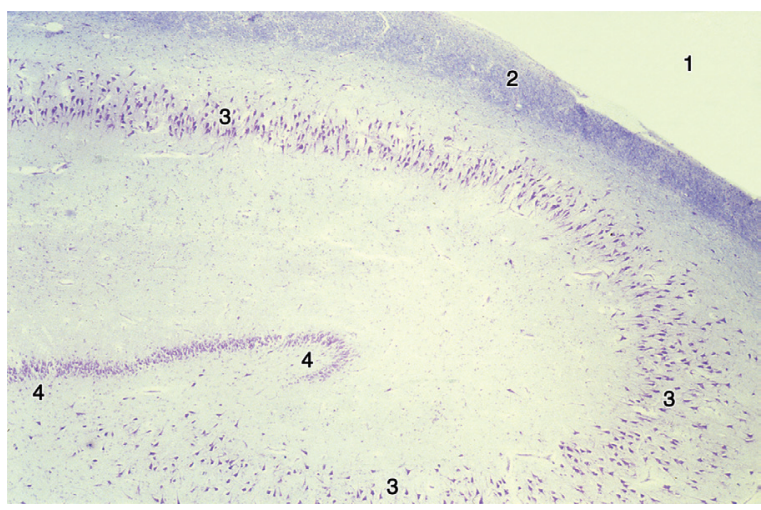

Figure 2. Coronal section of the left hippocampus; 1 - the inferior horn of the lateral ventricle; 2 - the alveus; 3 - the pyramidal neurons of the hippocampus; 4 - the dentate gyrus. Cresyl violet staining.

There was no significant difference of the HF volume in the elderly left-handed volunteers related to the right and left hemispheres $(t=1.22$; $D F=8$; $p>0.05)$, neither in the right-handed individuals related to the 2 hemispheres $(t=1.52 ; D F=20$; $p>0.05)$. On the other hand, the right-to-left HF 

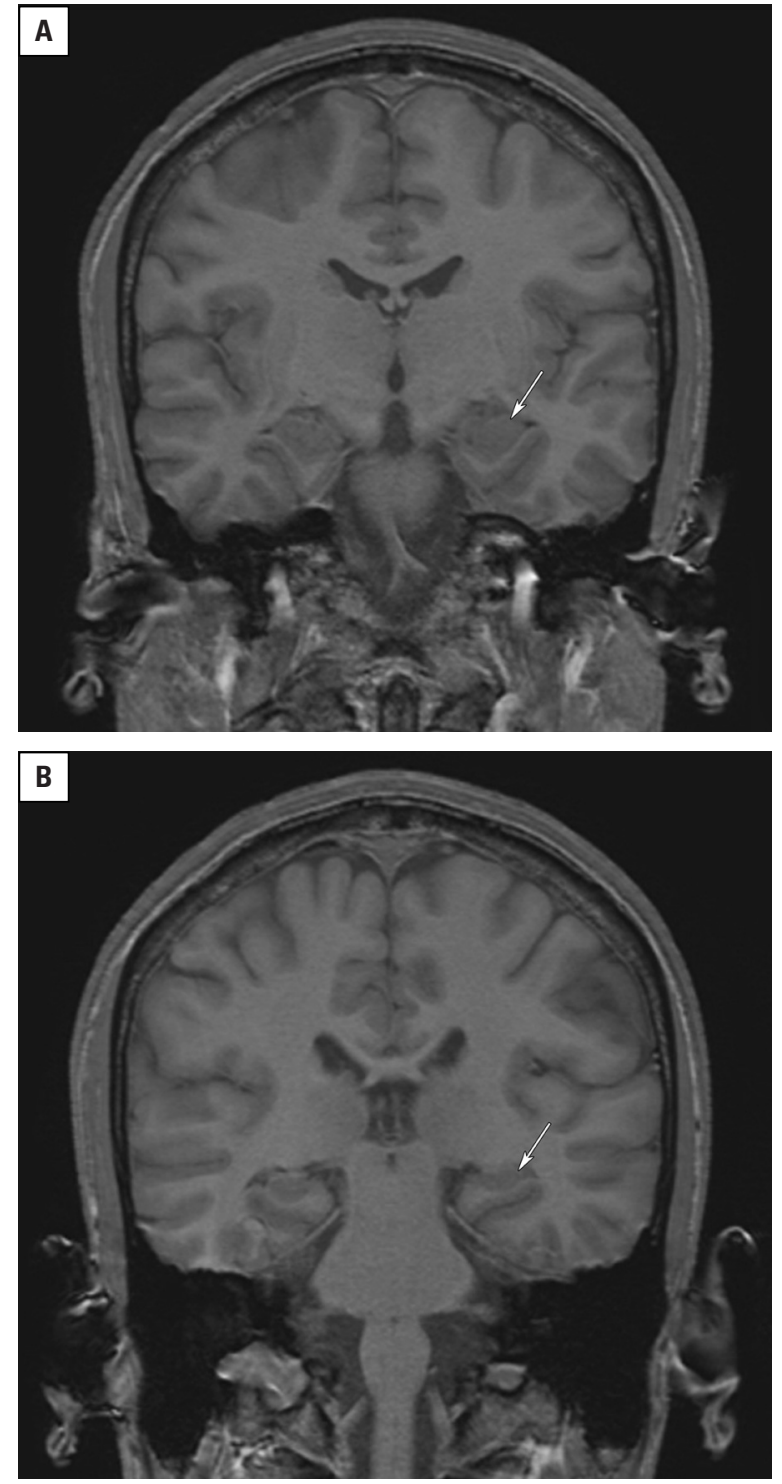

Figure 3. A coronal 3 tesla magnetic resonance imaging section through the anterior (A) and posterior (B) part of the hippocampal formation (arrow).

volume ratios differed significantly between the rightand left-handed participants $(p<0.05)$. Although there are also differences in the corresponding $\mathrm{HF}$ volumes between the left- and right-handed persons, they were not statistically significant.

We found a significant difference of the left HF volume between the young and elderly left-handed volunteers $(t=5.42 ; D F=29 ; p<0.01)$, as well as of the right $\mathrm{HF}$ volume between the young and the elderly left-handed volunteers $(t=4.88, \mathrm{DF}=29$, $p<0.01$ ). In addition, we also noticed a significant difference of the left HF volume of the young and aged right-handed volunteers $(t=6.92 ; \mathrm{DF}=29$; $p<0.01)$. Finally, a significant difference of the right HF volume was noticed between the young and elderly right-handed participants $(t=7.58 ; \mathrm{DF}=29 ; \mathrm{p}<0.01)$. All in all, significant differences were revealed of the $\mathrm{HF}$ volume on both sides between the two groups of various ages, meaning that the volume of the HF becomes reduced with age.

\section{DISCUSSION}

There are certain difficulties in the obtaining and evaluation of the volumetric findings in modern imaging techniques. Firstly, the MR equipment and the used imaging parameters differ among the institutions, for example the strength of the magnetic field, the pulse sequence and the slice thickness $[5,16,24$, $27,34]$. Secondly, the application of various methods for the HF delineation, either the manual tracing or the automatic segmentation, may influence the final result as well $[30,36,46,47]$. Thirdly, the brain size of the participants exerts substantial statistical influences on the HF volume values [4]. To avoid the latter, we applied the described normalisation of the HF volume. Finally, the determination of the HF borders is also important, so that various protocols have been used to overcome that problem $[4,16]$.

As regards the mean value of the HF volume, different authors mentioned the values as ranging between $2.78 \mathrm{~cm}^{3}$ and $3.91 \mathrm{~cm}^{3}$ [36], which is in accordance with our results (Table 1). However, there

Table 1. Normalised average volumes of the hippocampal formation regarding handedness and age

\begin{tabular}{|c|c|c|c|c|}
\hline \multirow[t]{2}{*}{ Ages } & \multirow[t]{2}{*}{ Subjects } & \multicolumn{2}{|c|}{ Normalised HF volumes $\left[\mathrm{cm}^{3}\right]$} & \multirow{2}{*}{$\begin{array}{l}\text { Right-to-left HF volume } \\
\text { ratios }\end{array}$} \\
\hline & & Right HF & Left HF & \\
\hline \multirow[t]{2}{*}{$22 \pm 2$} & $\mathrm{RH}$ & $2.986 \pm 265$ & $2.858 \pm 278$ & $1.03 \pm 0.06$ \\
\hline & LH & $2.879 \pm 309$ & $3.020 \pm 338$ & $0.95 \pm 0.05$ \\
\hline \multirow[t]{2}{*}{$75 \pm 2$} & $\mathrm{RH}$ & $2.728 \pm 248$ & $2.650 \pm 256$ & $1.02 \pm 0.05$ \\
\hline & $\mathrm{LH}$ & $2.617 \pm 220$ & $2.780 \pm 250$ & $0.94 \pm 0.04$ \\
\hline
\end{tabular}

HF — hippocampal formation; RH — right-handed; LH — left-handed 
are certain racial and national differences. For example, the mean $\mathrm{HF}$ volume within the Indian population is $2.411 \mathrm{~cm}^{3}$, with the mean values for the right $\mathrm{HF}$ as $2.424 \mathrm{~cm}^{3}$ and for the left $\mathrm{HF}$ as $2.398 \mathrm{~cm}^{3}$ [31]. The range in the Chinese people is $2.204-2.944 \mathrm{~cm}^{3}$ for the right $\mathrm{HF}$, and $2.068-2.700 \mathrm{~cm}^{3}$ for the left $\mathrm{HF}$ [27]. The values in both populations are smaller than in the Caucasians. For example, Honeycutt and Smith [17] reported $2.90 \mathrm{~cm}^{3}$ on average on the right side and $2.78 \mathrm{~cm}^{3}$ on the left side, whereas Pruessner et al. [36] mentioned $3.324 \mathrm{~cm}^{3}$ for the right $\mathrm{HF}$, and $3.208 \mathrm{~cm}^{3}$ for the left HF, respectively. Some other authors also found a larger right HF volume [18, 29, 34].

The larger values for the right than the left HF volume were also noticed in our study, that is $2.986 \mathrm{~cm}^{3}$ : $2.858 \mathrm{~cm}^{3}$ in the young group and $2.728 \mathrm{~cm}^{3}: 2.650 \mathrm{~cm}^{3}$ in the aged group, but only in the right-handed individuals (Table 1). There was a quite opposite situation with the left-handed participants of both groups, i.e. $2.879 \mathrm{~cm}^{3}: 3.020 \mathrm{~cm}^{3}$ volume of the left HF among the young volunteers, and $2.617 \mathrm{~cm}^{3}: 2.780 \mathrm{~cm}^{3}$ among the elderly participants. However, those results did not show a statistical significance.

The young left-handed participants in our study showed greater left than right HF volume ratios (Table 1). Although the mean value of the right $\mathrm{HF}$ volume was larger than the left HF volume among the right-handed young participants, this difference was not statistically significant. Nonetheless, the statistical difference was noted for the left HF volume ratios between the right- and left-handed individuals, but not for the right HF in the same aged group. Szabo et al. [44] found that only in the right-handed participants the HF was significantly larger on the right side, whereas the left-handed group did not show a significant difference between the right and left HF. The right-to-left $\mathrm{HF}$ volume ratios differed significantly between the right- and left-handed volunteers in their study.

Nevertheless, such right-left differences mostly develop in utero under the influence of certain genes [39]. In general, the right-left asymmetry of the $\mathrm{HF}$, related to the handedness, is only part of the global hemispheric asymmetry, which is based on the various structural and functional features of the corresponding right and left regions of the brain [12, $21,23,40]$. We cannot explain the "inversion" of the $\mathrm{HF}$ asymmetry, for instance, a slightly larger right HF volume in the right-handed volunteers, whose left hemisphere is dominant for the hand motor skills [12].
Yet, the left hippocampus in the right-handed persons seems to have a crucial role in language acquisition, development and word memory [23].

In our study, the influence of age could be expressed as a smaller HF volume, both in the left and right hemispheres and in both the left- and right-handed elderly participants. Some authors have also drawn a special attention to the $\mathrm{HF}$ in elderly healthy people $[8,33,35,43,48]$. A few of them noticed a progressive reduction (from $1.18 \%$ to $2.8 \%$ per year of age) of the $\mathrm{HF}$ volume in persons over the age of 65 years. Some of the other authors revealed a correlation between the $\mathrm{HF}$ reduction and cognitive decline [49].

Certain authors noticed a reorganisation of the hippocampal neural network supporting normal cognitive function in old people [8], whilst some others suggested that the process of aging affects mainly the metabolic status of the hippocampus, with little equivalent age-related changes in the hippocampal cell density [45]. Anyway, certain brain tissue reduction, predominantly grey matter rather than white matter, is a common feature of the normal aging process. Regardless of the mentioned annual amount of reduction, the neuronal loss in healthy old people is always less than $10 \%$ [32]. Finally, some lifestyle factors, in association with the expression of certain genes, promote a healthy and successful aging of the brain and delay the appearance of age-related cognitive deficits in elderly people [32].

As regards the relationship between the HF asymmetry and gender of the participants, different data can be found in the literature. Thus, Jiang et al. [21] concluded that there could be a relationship between the HF volume, gender and certain aspects of memory. According to Maller et al. [29], the right $\mathrm{HF}$ volume is somewhat larger in man, but in the next paper [28] they reported that it was larger in both genders. Anstey et al. [2] found that the HF volumes were larger in the older left-handed women than in the right-handed ones, which they interpreted as one of the explanations for lower rates of dementia in the left-handed individuals. However, there was no association with handedness in men, nor right-left asymmetry in the HF volume in either gender. According to some others [11, $25]$, the brain size is more important than sex in explaining interindividual differences in volume of its parts. In any case, the eventual presence of the $\mathrm{HF}$ asymmetry in general population, as was noticed in our study, can be explained by the influence of 
corresponding $\mathrm{X}$ chromosome genes and the sex hormones [15, 39].

Certain morphometric HF abnormalities were found in some psychiatric patients, for instance in those with schizophrenia $[13,37]$. The HF changes in volume were also seen in patients with cognitive impairment, including those with Alzheimer's disease $[1,46]$. Reduction in the HF volume was observed in heavy cannabis use, in epilepsy, mild cognitive decline, dementia, major depression, the aged, traumatic brain injury, posttraumatic stress disorder, chronic alcoholism, borderline and antisocial personality disorders, obsessive-compulsive disorder, herpes simplex encephalitis, Parkinson's and Huntington's disease, Down's and Turner's syndrome, cardiac arrest, and Cushing's disease $[1,10,14,38,42]$.

Asymmetric damage of the hippocampus, for example a smaller $\mathrm{HF}$ volume on one side, is seen in the chronic stress condition, anxiety, sleep disturbance, impaired cognitive functions etc. [22, 26, 41]. The hippocampal atrophy is correlated with Alzheimer's disease and mild cognitive impairment for the right hippocampus [49]. Some authors found in schizophrenic patients certain neurochemical lateralisation in the $\mathrm{HF}$ in relation to quantity of the glutamate receptors and size of the pyramidal cell synapses [13, 40]. Finally, functional or pathophysiological right-left $\mathrm{HF}$ asymmetry was noticed in memory processing [9], language acquisition [23], posttraumatic stress disorders [41, 48], and cognitive decline of various causes [10].

\section{CONCLUSIONS}

We did not find a significant difference in the HF volume related to the side among the right-handed young participants, but we noticed a statistically significant difference in the HF volume ratio among the left-handed young volunteers. The HF volume in both hemispheres among the left- and right- handed persons becomes reduced over years. In other words, certain atrophy of both the HFs was present in the elderly group. It is obvious that he MRI study can be a sophisticated and reliable method in volumetric analysis of the hippocampal formation in various domains.

\section{ACKNOWLEDGEMENTS}

This work was supported by the grant No.143016 from the Ministry of Science of Serbia.
We are very grateful to Mrs. Elza Holt for reviewing the English text of our manuscript

\section{REFERENCES}

1. Anstey KJ, Maller JJ (2003) The role of volumetric MRI in understanding mild cognitive impairment and similar classifications. Aging Ment Health, 7: 238-250.

2. Anstey KJ, Maller JJ, Meslin C, Christensen H, Jorm AF, Wen W, Sachdev P (2004) Hippocampal and amygdalar volumes in relation to handedness in adults aged 60-64. Neuroreport, 15: 2825-2829.

3. Bhatia S, Bookheimer SY, Gaillard WD, Theodore WH (1993) Measurement of whole temporal lobe and hippocampus for MR volumetry: normative data. Neurology, 43: 2006-2010.

4. Bilir E, Craven W, Hugg J, Gilliam F, Martin R, Faught E, Kuzniecky R (1998) Volumetric MRI of the limbic system: anatomic determinants. Neuroradiology, 40: 138-144.

5. Briellmann RS, Syngeniotis A, Jackson GD (2001) Comparison of hippocampal volumetry at 1.5 tesla and at 3 tesla. Epilepsia, 42: 1021-1024.

6. Carpenter MB (2001) Core text of neuroanatomy. Williams \& Wilkinson, Baltimore.

7. Cendes F, Leproux F, Melanson D, Sharbrough FW, Hirschorn KA, Meyer FB, Marsh WR, O'Brien PC (1993) Magnetic resonance imaging-based volume studies in temporal lobe epilepsy: pathological correlations. Comput Assist Tomogr, 17: 206-210.

8. Crivello F, Tzourio-Mazoyer N, Mazoyer B (2014) Longitudinal assesment of global and regional rate of grey matter atrophy in 1,172 healthy older adults: modulation by sex and age. PloS One, 9: e114478.

9. Cuzzocreo JL, Yassa MA, Verduzco G, Honeycut NA, Scott DJ, Bassett SS (2009) Effect of handedness on fMRI activation in the medial temporal lobe during an auditory verbal memory task. Hum Brain Mapp, 30: 1271-1278.

10. Elcombe EL, Lagopoulos J, Duffy S, Lewis SJ, Norrie L, Hickle IB, Naismith SL (2015) Hippocampal volume in older adults at risk of cognitive decline: the role of sleep, vascular risk, and depression. J Alzheimer's Dis, 44: 1279-1290.

11. Free SL, Bergin PS, Fish DR, Cook MJ, Shorvon SD, Stevens JM (1995) Methods for normalization of hippocampal volumes measured with MR. Am J Neuroradiol, 4: 637-643.

12. Galaburda AM, Rosen GD, Sherman GF (1990) Individual variability in cortical organization: its relationship to brain laterality and implications to function. Neuropsychologia, 28: 529-546.

13. Geddes AE, Huang XF, Newell KA (2014) GluN2B protein deficits in the left, but not the right, hippocampus in schizophrenia. BMC Psychiatry, 8: 274-281.

14. Geuze E, Vermetten E, Bremner JD (2005) MR-based in vivo hippocampal volumetrics: 2 . Findings in neuropsychiatric disorders. Mol Psychiatry, 10: 160-184.

15. Giedd JN, Vaituzis AC, Hamburger SD, Lange N, Rajapakse JC, Kaysen D, Vauss YC, Rapoport JL (1996) Quantitative MRI of the temporal lobe, amygdala, and hippocampus in normal human development: ages $4-18$ years. J Comp Neurol, 366: 223-230. 
16. Helenius J, Soinne L, Perkiö J, Salonen O, Kangasmäki A, Kaste M, Carano RA, Aronen HJ, Tatlisumak T (2002) Diffusion-weighted MR imaging in normal human brains in various age groups. Am J Neuroradiol, 23: 194-199.

17. Honeycutt NA, Smith CD (1995) Hippocampal volume measurements using magnetic resonance imaging in normal young adults. J Neuroimaging, 5: 95-100.

18. Horváth K, Kövér F, Kovács N, Kállai J, Nagy F (2002) Volumetric measurements of the hippocampus and amygdala with MRI in healthy adults. Orv Hetil, 143: 2145-2151.

19. Immordino-Yang MH, Singh V (2103) Hippocampal contributions to the processing of social emotions. Hum Brain Mapp, 34: 945-955.

20. Inskip PD, Tarone RE, Brenner AV, Fine HA, Black PM, Shapiro WR, Selker RG, Linet MS (2003) Handedness and risk of brain tumors in adults. Cancer Epidemiol Biomarkers Prev, 12: 223-225.

21. Jiang L, Cheng Y, Li Q, Tang Y, Shen Y, Li T, Feng W, Cao X, Wu W, Wang J, Li C (2014) Cross-sectional study of the association of cognitive function and hippocampal volume among healthy elderly adults. Shanghai Arch Psychiatry, 26: 280-287.

22. Joo EY, Kim H, Suh S, Hong SB (2014) Hippocampal substructural vulnerability to sleep disturbance and cognitive impairment in patients with chronic primary insomnia: magnetic resonance imaging morphometry. Sleep, 37: 1189-1198.

23. Knecht S (2004) Does language lateralization depends on the hippocampus? Brain, 127: 1217-1218.

24. Laakso MP, Juottonen K, Partanen K, Vainio P, Soininen H (1997) MRI volumetry of the hippocampus: the effect of slice thickness on volume formation. Magn Reson Imag, 15: 263-265.

25. Lancaster JL, Glass TG, Lankipalli BR, Downs H, Mayberg H, Fox PT (1996) A modality-independent approach to spatial normalization of tomography images of the human brain. Hum Brain Mapp, 3: 209-223.

26. Levita L, Bois C, Healey A, Smyllie E, Papakonstantinou E, Hartley T, Lever C (2014) The behavioural inhibition system, anxiety and hippocampal volume in a non-clinical populatiion. Biol Mood Anxiety Disord, 7: 4-12.

27. Li YJ, Ga SN, Huo Y, Li SY, Gao XG (2007) Characteristics of hippocampal volumes in healthy Chinese from MRI. Neurol Res, 29: 803-806.

28. Maller JJ, Anstey KJ, Réglade-Meslin C, Christensen $\mathrm{H}_{\text {, }}$ Wen W, Sachdev P (2007) Hippocampus and amygdala volumes in a random community-based sample of 60-64 year olds and their relationship to cognition. Psychiatry Res, 156: 185-197.

29. Maller JJ, Réglade-Meslin C, Anstey KJ, Sachdev P (2006) Sex and symmetry differences in hippocampal volumetrics: before and beyond the opening of the crus of the fornix. Hippocampus, 16: 80-90.

30. McHugh TL, Saykin AJ, Wishart HA, Flashman LA, Cleavinger HB, Rabin LA, Mamourian AC, Shen L (2007) Hippocampal volume and shape analysis in an older adult population. Clin Neuropsychol, 21: 130-145.

31. Mohandas AN, Bharath RD, Prathyusha PV, Gupta AK (2014) Hippocampal volumetry: Normative data in the Indian population. Ann Indian Acad Neurol, 17: 267-271.

32. Mora F (2013) Successful brain aging: plasticity, environmental enrichment, and lifestyle. Dialogues Clin Neurosci, 15: 45-52.

33. Mu Q, Xie J, Wen Z, Weng Y, Shuyun Z (1999) A quantitative MR study of the hippocampal formation, the amygdala, and the temporal horn of the lateral ventricle in healthy subjects 40 to 90 years of age. Am J Neuroradiol, 20: 207-211.

34. Pedraza O, Bowers D, Gilmore R (2004) Asymmetry of the hippocampus and amygdala in MRI volumetric mea- surements of normal adults. J Int Neuropsychol Soc, 10: 664-678.

35. Petersen RC, Jack CR Jr, Xu YC, Waring SC, O'Brien PC, Smith GE, Ivnik RJ, Tangalos EG, Boeve BF, Kokmen E (2000) Memory and MRI-based hippocampal volumes in aging and AD. Neurology, 54: 581-587.

36. Pruessner JC, Li LM, Serles W, pruessner M, Collins DL, Kabani N, Lupien S, Evans AC (2000) Volumetry of hippocampus and amygdala with high-resolution MRI and three-dimensional analysis software: minimizing the discrepancies between laboratories. Cereb Cortex, 10: 433-442.

37. Pujol $N$, Penadés $R$, Junqué $C$, Dinov I, Fu $C H$, Catalán $R$, Ibarretxe-Bilbao N, Bargalló N, Bernardo M, Toga A, Howard RJ, Costafreda SG (2014) Hippocampal abnormalities and age in chronic schizophrenia: morphometric study across the adult lifespan. Br J Psychiatry, 205: 369-375.

38. Ramezani M, Johnsrude I, Rasoulian A, Bosma R, Tong R, Hollenstein T, Harkness K, Abolmaesumi P (2014) Temporal-lobe morphology differs beween healthy adolescents and those with early-onset of depression. Neuroimage Clin, 14: 145-155.

39. Savic I (2014) Asymmetry of cerebral gray and white matter and structural volumens in relation to sex hormones and chromosomes. Front Neurosci, 8: 1-13.

40. Shinohara $Y$, Hirase $H$, Watanabe $M$, Itakura $M$, Takahashi M, Shigemoto R (2014) Left-right asymmetry of the hippocampal synapses with differential subunit allocation of glutamate receptors. Proc Natl Acad Sci, 105: 19498-19503.

41. Shu XJ, Xue L, Liu W, Chen FY, Zhu C, Sun $X H$, Wang $X P$, Liu ZC, Zhao H (2013) More vulnerability of left hand right hippocampal damage in right-handed patients with post-traumatic stress disorder. Psychiatry Res, 212: 237-244.

42. Sivakumar PT, Kalmady SV, Venkatasubramanian G, Bharath S, Reddy NN, Rao NP, Kovoor JM, Jain S, Varghese M (2015) Volumetric analysis of hippocampal sub-regions in late onset depression: A 3 tesla magnetic resonance imaging study. Asian J Psychiatr, 13: 38-43.

43. Sullivan EV, Marsh L, Pfefferbaum A (2005) Preservation of hippocampal volume throughout adulthood in healthy men and women. Neurobiol Aging, 26: 1093-1098.

44. Szabo A, Xiong J, Lancaster J, Rainey L, Fox P (2001) Amygdalar and hippocampal volumetry in control participants: differences regarding handedness. Am J Neuroradiol, 22: 1342-1345.

45. Szenkuti A, Guderian S, Schiltz K, Kaufmann J, Münte TF, Heinze HJ, Düzel E (2004) Quantitative MR analyses of the hippocampus: unspecific metabolic changes in aging. J Neurol, 25: 1345-1353.

46. Voevodskaya O, Simmons A, Nordenskjöld R, Kullberg J, Ahiström H, Lind L, Wahlund LO, Larsson EM, Westman E (2014) The effects of intracranial volume adjustment approaches on multiple regional MRI volumes in healthy aging and Alzheimer's disease. Front Aging Neurosci, 6: 264-272.

47. Wenger $E$, Mårtensson J, Noack H, Bodammer NC, Kühn S, Schaefer S, Heinze HJ, Düzel E, Bäckman L, Lindenberger U, Lövdén M (2014) Comparing manual and automatic segmentation of hippocampal volumes: reliability and validity issues in younger and older brains. Hum Brain Mapp, 35: 4236-4248.

48. Woon FL, Sood S, Hedges DW (2010) Hippocampal volume deficits associated with exposure to psychological trauma and posttraumatic stress disorder in adults: a meta-analysis. Prog Neuropsychopharmacol Biol Psychiatry, 34: 1181-1188.

49. Yavuz BB, Ariogul S, Cankurtaran M, Oguz KK, Halil M, Dagli N, Cankurtaran ES (2007) Hippocampal atrophy correlates with the severity of cognitive decline. Int Psychogeriatr, 19: 767-777. 